\title{
Adolescent subthreshold-depression and anxiety: psychopathology, functional impairment and increased suicide risk
}

\author{
Judit Balázs, ${ }^{1,2}$ Mónika Miklósi, ${ }^{3,4}$ Ágnes Keresztény, 1,3 Christina W. Hoven, 5,6 \\ Vladimir Carli, ${ }^{7}$ Camilla Wasserman, ${ }^{5,8}$ Alan Apter, ${ }^{9}$ Julio Bobes, ${ }^{10}$ Romuald \\ Brunner, ${ }^{11}$ Doina Cosman, ${ }^{12}$ Pádraig Cotter, ${ }^{13}$ Christian Haring, ${ }^{14}$ Miriam \\ Iosue, ${ }^{8}$ Michael Kaess, ${ }^{11,15}$ Jean-Pierre Kahn, ${ }^{16}$ Helen Keeley, ${ }^{13}$ Dragan \\ Marusic, ${ }^{17}$ Vita Postuvan, ${ }^{17}$ Franz Resch, ${ }^{11}$ Pilar A Saiz, ${ }^{10}$ Merike Sisask, ${ }^{18}$ \\ Avigal Snir, ${ }^{9}$ Alexandra Tubiana, ${ }^{16}$ Airi Varnik, ${ }^{18}$ Marco Sarchiapone, ${ }^{8}$ and \\ Danuta Wasserman
}

\begin{abstract}
${ }^{1}$ Vadaskert Child and Adolescent Psychiatric Hospital, Budapest, Hungary; ${ }^{2}$ Institute of Psychology, Eötvös Loránd University, Budapest, Hungary; ${ }^{3}$ Semmelweis University, School of Ph.D. Studies, Budapest, Hungary; ${ }^{4}$ Heim Pál Paediatric Hospital, Budapest, Hungary; ${ }^{5}$ Department of Child and Adolescent Psychiatry, Columbia University-New York State Psychiatric Institute, New York, NY, USA; ${ }^{6}$ Department of Epidemiology, Mailman School of Public Health, Columbia University, New York, NY, USA; ${ }^{7}$ National Centre for Suicide Research and Prevention of Mental Ill-Health (NASP), Karolinska Institute, Stockholm, Sweden; ${ }^{8}$ Medicine and Health Science Department, University of Molise, Campobasso, Italy; ${ }^{9}$ Feinberg Child Study Center, Schneider Children's Medical Center, Tel Aviv University, Tel Aviv, Israel; ${ }^{10}$ Department of Psychiatry, School of Medicine, University of Oviedo; Centro de InvestigaciónBiomédica en Red de Salud Mental, CIBERSAM, Oviedo, Spain; ${ }^{11}$ Section Disorders of Personality Development, Clinic of Child and Adolescent Psychiatry, University of Heidelberg, Heidelberg, Germany; ${ }^{12}$ Clinical Psychology Department, Iuliu Hatieganu University of Medicine and Pharmacy, Cluj-Napoca, Romania; ${ }^{13}$ National Suicide Research Foundation, Cork, Ireland; ${ }^{14}$ Research Division for Mental Health, University for Medical Information Technology (UMIT), Innsbruck, Austria; ${ }^{15}$ Orygen Youth Health Research Centre, The University of Melbourne, Melbourne, Vic., Australia; ${ }^{16}$ Department of Psychiatry, Centre Hospitalo-Universitaire (CHU) de NANCY, Université H. Poincaré, NANCY-France; ${ }^{17}$ Mental Health Department, PINT, University of Primorska, Primorska, Slovenia; ${ }^{18}$ EstonianSwedish Mental Health \& Suicidology Institute, Ctr. Behav \& Hlth Sci, Tallinn University, Tallinn, Estonia;
\end{abstract}

\begin{abstract}
Background: Subthreshold-depression and anxiety have been associated with significant impairments in adults. This study investigates the characteristics of adolescent subthreshold-depression and anxiety with a focus on suicidality, using both categorical and dimensional diagnostic models. Methods: Data were drawn from the Saving and Empowering Young Lives in Europe (SEYLE) study, comprising 12,395 adolescents from 11 countries. Based on self-report, including Beck Depression Inventory-II (BDI-II), Zung Self-Rating Anxiety Scale (SAS), Strengths and Difficulties Questionnaire (SDQ) and Paykel Suicide Scale (PSS) were administered to students. Based on BDI-II, adolescents were divided into three groups: nondepressed, subthreshold-depressed and depressed; based on the SAS, they were divided into nonanxiety, subthreshold-anxiety and anxiety groups. Analyses of Covariance were conducted on SDQ scores to explore psychopathology of the defined groups. Logistic regression analyses were conducted to explore the relationships between functional impairments, suicidality and subthreshold and full syndromes. Results: Thirty-two percent of the adolescents were subthreshold-anxious and 5.8\% anxious, $29.2 \%$ subthreshold-depressed and 10.5\% depressed, with high comorbidity. Mean scores of SDQ of subthreshold-depressed/anxious were significantly higher than the mean scores of the nondepressed/nonanxious groups and significantly lower than those of the depressed/anxious groups. Both subthreshold and threshold-anxiety and depression were related to functional impairment and suicidality. Conclusions: Subthreshold-depression and subthreshold-anxiety are associated with an increased burden of disease and suicide risk. These results highlight the importance of early identification of adolescent subthreshold-depression and anxiety to minimize suicide. Incorporating these subthreshold disorders into a diagnosis could provide a bridge between categorical and dimensional diagnostic models. Keywords: Categorical diagnostic model, dimensional diagnostic model, subthreshold-depression, subthreshold-anxiety, adolescent, suicide, SEYLE.
\end{abstract}

\section{Introduction}

There is mounting criticism of the current classification systems (Diagnostic and Statistical Manual of Mental Disorders Text Revised (DSM-IV-TR) (American

Conflicts of interest statement: No conflicts declared.
Psychiatric Association, 2000) and International Classification of Mental and Behavioral Disorders (ICD-10) (World Health Organization, 1992), with increasing evidence for the advantages and disadvantages of both categorical and dimensional approaches (Lecrubier, 2008; Möller, 2008; Okasha, 2009). Individuals requiring psychiatric intervention may not receive a standard diagnosis based on the 
DSM-IV-TR or ICD-10 due to an insufficient number or duration of symptoms (Johnson, Weissman, \& Klerman, 1992). Patients with substantial functional impairment who do not meet diagnostic criteria are regarded as having subthreshold disorders (Judd, Rapaport, Paulus, \& Brown, 1994). Helmchen and Linden (2000) suggest that subthreshold diagnoses are not solely artefacts from potentially outdated definitions, but rather unique conditions demanding recognition. It has been suggested that implementing a hybrid of categorical and dimensional approaches in DSM-V would be useful, as both are important for clinical work and research (Okasha, 2009).

A large number of studies have focused on child and adolescent subthreshold-depression (Fergusson, Horwood, Ridder, \& Beautrais, 2005; Foley, Goldston, Costello, \& Angold, 2006; Keenan et al., 2008; Klein, Shankman, Lewinsohn, \& Seeley, 2009; Lewinsohn, Solomon, Seeley, \& Zeiss, 2000), showing that subthreshold-depression increases the risk of developing a major depressive episode (MDE) (Pine, Cohen, Cohen, \& Brook, 1999; Shankman et al., 2009). Although the high comorbidity of anxiety [specifically generalized anxiety disorder (GAD)] and MDE are well described (Kessler, Chiu, Demler, Merikangas, \& Walters, 2005; Unick, Snowden, \& Hastings, 2009; Wittchen, Zhao, Kessler, \& Eaton, 1994), there are still few studies on subthreshold-GAD among children/adolescents (Foley et al., 2006; Guberman \& Manassis, 2011; Nauta et al., 2012).

Epidemiological data on child/adolescent subthreshold-depression vary, with 12-month prevalence ranging from $3 \%$ to $12 \%$, and lifetime prevalence through late adolescence as high as $26 \%$ (Fergusson et al., 2005; Wittchen, Nelson, \& Lachner, 1998). To our knowledge, no study has examined the prevalence of subthreshold-GAD among youth, whereas among adults, the 12-month prevalence was found to be 3.6-15.7\% (Carter, Wittchen, Pfister, \& Kessler, 2001; Rucci et al., 2003).

This variability in epidemiological data may be explained, in part, by different definitions and diagnostic methodologies. Some studies used standardized clinical interviews to screen for subthresholddepression and subthreshold-GAD (Carter et al., 2001; Fergusson et al., 2005; Foley et al., 2006; Guberman \& Manassis, 2011; Keenan et al., 2008; Rucci et al., 2003; Shankman et al., 2009), others used self-report (Lewinsohn et al., 2000) or both (Nauta et al., 2012).

Broad and narrow definitions of child/adolescent subthreshold-depression and subthreshold-GAD exist with respect to both the number and the duration of symptoms and additional criteria (e.g. presence of distress), but there is no accepted definitions of these conditions (Angst, Merikangas, \& Preisig, 1997; Fergusson et al., 2005; Foley et al., 2006; Karsten, Nolen, Penninx, \& Hartman, 2011; Keenan et al., 2008; Kertz \& Woodruff-Borden,
2011; Klein et al., 2009; Rucci et al., 2003; Shankman et al., 2009).

Psychiatric disorders, especially MDE, are major risk factors for suicidal behaviour (Gould et al., 1998). Comorbidity, mainly anxiety disorders, increases the risk of suicidal behaviour among adolescents (Wunderlich, Bronisch, \& Wittchen, 1998). Balázs, Bitter, Lecrubier, Csiszér, and Ostorharics (2000) found that almost two thirds of adult suicide attempters had MDE, half had GAD, one tenth had subthreshold-depressive episode and one fifth had subthreshold-GAD. Only a few adolescent studies have focused on subthreshold mental disorders, including subthreshold-depressive episodes and GAD, as a possible risk factor for suicide. Foley et al. (2006) examined subjects aged 9-16 years and found that suicidal youth without a full DSM-IV-TR; psychiatric disorder had significantly higher prevalence of subthreshold conditions than nonsuicidal youth without psychiatric disorders.

The aim of the present study of European adolescents was the examination of the prevalence of subthreshold-depression and subthreshold-anxiety and its relationships with psychopathology, functional impairment and suicidal behaviour.

\section{Method \\ Participants}

The sampling procedures of the Saving and Empowering Young Lives in Europe (SEYLE) study were previously described (Wasserman et al., 2010). SEYLE's sample of 12,395 adolescents (aged 14-16 years) is from 11 European countries: Austria, Estonia, France, Germany, Hungary, Ireland, Israel, Italy, Romania, Slovenia and Spain, with Sweden serving as the coordinating centre. Ethical approval was obtained from each site's local ethics committee. Local school authorities granted access to randomly selected school(s) and informed assent and consent were obtained, as required.

\section{Data collection}

Students were administered a self-report questionnaire that included well-established measures and items developed for SEYLE (Wasserman et al., 2010).

Beck Depression Inventory-II (BDI-II) measured severity of depression by assessing specific symptoms experienced over the preceding 2 weeks (Beck, Steer, Ball, \& Ranieri, 1996; Byrne, Stewart, \& Lee, 2004). BDI-II item 'loss of interest in sex' was excluded from the SEYLE version because it was considered inappropriate in some cultural settings (Byrne et al., 2004).

Symptoms of current anxiety were assessed using Zung Self-Rating Anxiety Scale (SAS) (Zung, 1971), a 20-item self-report questionnaire. Zung referred to the scores as an 'Index score' ('normal range': $\leq 44$; 'minimal to moderate anxiety': 45-59; 'marked to severe anxiety': 60-74; 'extreme anxiety': 275) (McDowell, 2006).

Psychopathology was evaluated using Strengths and Difficulties Questionnaire (SDQ), a brief instrument for 
screening childhood behaviours, consisting of 25 items (Goodman, Meltzer, \& Bailey, 1998). The extended version of the SDQ was used, which includes an impact supplement, a measure of functional impairment.

Frequency of suicidal thoughts/ideations was assessed during the past 2 weeks using Paykel Suicide Scale (PSS) (Paykel, Myers, Lindenthal, \& Tanner, 1974).

\section{Definitions}

Adolescents were divided into three groups based on BDI-II score: $\geq 20$ = depressed (Beck et al., 1996); <20 (BDI-II) and being positive $(>0)$ on items assessing core symptoms of DSM-IV-TR MDE (sadness or loss of pleasure $)=$ subthreshold-depression; all others $=$ nondepressed.

Adolescents were also divided into three groups based on the SAS: $\geq 60=$ anxious; $45 \leq$ and $<60=$ subthreshold-anxious; $<45=$ nonanxious.

\section{Analyses}

Data were analysed using IBM SPSS Statistic 20 software package (SPSS, Inc, 2011). Gender differences among nondepressed, subthreshold and depressed, as well as nonanxious, subthreshold and anxious groups were analysed using chi-squared tests. One-way ANOVAs were conducted to explore age differences. Means and standard deviations, as well as percentages of borderline and elevated SDQ total and impact scores are reported for the three groups of anxiety/depression, using the cut-off defined by Goodman et al. (1998). Due to cross-cultural variation in cut-off scores of the SDQ (Vostanis, 2006) continuous scores were used to explore levels of overall psychopathology. Bivariate relationships between measures of anxiety and depression, and SDQ total score were explored using Spearman correlations. Analyses of Covariance (ANCOVA) with Tukey post hoc test were conducted separately for girls and boys with age as covariate to detect differences among the three study groups. To differentiate the effects of anxiety and depression, each analysis was controlled for by depression/anxiety (for BDI-II total score when exploring the effect of subthreshold and full anxiety on functional impairments and for SAS total score when the effects of subthreshold and full depression were studied). Logistic regression analyses with dummy-coded SDQ impact score (0 'normal' and $0<$ 'borderline/elevated scores') and PSS total score (0 or greater) as independents, were conducted to explore the relationships between functional impairments, as well as suicidality and subthreshold and full anxiety/ depression. All analyses were adjusted for gender, age and continuous score of anxiety/depression. Due to sensitivity of Hosmer and Lemeshow goodness of fit test for very large sample sizes (Kramer \& Zimmerman, 2007), the area under the Receiver operating characteristic (ROC) curve [area under the roc curve (AUC)] was reported. A $p$-value of .05 was considered statistically significant. Effect size measures are also reported for all analyses.

Multiple imputations (MI) were conducted using the IBM SPSS Statistics 20 (2011) to account for missing data. Five imputed datasets were created. Variables included in the model: age, country of origin, gender, all
SDQ items, PSS, SAS and BDI-II as target variables and predictors. Each completed dataset was analysed using standard methods for assessing differences among nondepressed/nonanxious, subthreshold-depressed/anxious and depressed/anxious groups. Pooled estimates were calculated using Rubin's Rules (Rubin, 1987).

\section{Results \\ Subjects}

Complete data were obtained for 11,109 (89.6\%) of the 12,395 adolescents in SEYLE: 4,506 (40.7\%) boys and 6,565 (59.3\%) girls. Mean age: 14.80 years $(S D=.84)$.

Only $17,652(1.8 \%)$ of the total 961,553 data items were missing. The proportion of the missing data was greatest on the sixth item of the SDQ ('I am usually on my own. I generally play alone or keep to myself.') $(10.8 \%)$, whereas all other variables had less than $5 \%$ missing. Complete cases and incomplete cases differed significantly in age $(t(12310)=-48.356, p<$ .001 , Cohen's $d=1.384)$ and gender $\left(\chi^{2}(1)=755.363\right.$, $p<.001, \phi=-.248$ ), indicating that adolescents with incomplete data tended to be older and male subject.

MI analyses used data of all 12,395 participants: 5,529 males and 6,799 females (67 missing gender cases). The mean age was 14.91 years $(S D=.90)$.

\section{Anxiety and depression}

Among all 12,395 adolescents, 7,476 (60.3\%) were identified as nondepressed, 3,618 (29.2\%) subthreshold-depressed and 1,301 (10.5\%) depressed.

Analysis showed a significant age effect on group membership $(F(2)=28.321, p<.001)$. However, the effect size for this analysis $\left(\eta^{2}=.005\right)$ was not found to exceed Cohen's (1988) convention for a small effect $\left(\eta^{2}=.01\right)$.

Gender differences of small effect size were found among groups of nondepressed, subthreshold-depressed and depressed youth $\left(\chi^{2}(2)=254.956\right.$, $p<.001$, Cramer's $V=.143)$. Girls more frequently were both subthreshold-depressed and depressed (Table 1) $(p<.001, \phi=.106$ and .143 respectively).

Among all subjects, 7,708 (62.2\%) were identified as nonanxious, 3,964 (32.0\%) subthreshold-anxious and $723(5.8 \%)$ anxious.

Among the three levels of anxiety, a significant effect of group membership was found for age $(F(2)=44.846, p<.001)$. Again, the effect size $\left(\eta^{2}=.007\right)$ was not found to exceed Cohen's (1988) convention for a small effect.

Gender had a small effect on group membership across levels of anxiety $\left(\chi^{2}(2)=290.362, p<.001\right.$, Cramer's $V=.154)$. Girls more frequently were both subthreshold-anxious and anxious (Table 1) ( $p<.001, \phi=.115$ and .147 respectively).

Results revealed a strong relationship between SAS and BDI-II scores $(r=.503$ and .656 for boys 
Table 1 Gender distribution among nondepressed/nonanxious, subthreshold-depressed/anxious and depressed/anxious groups

\begin{tabular}{|c|c|c|c|c|}
\hline & \multicolumn{2}{|c|}{ Levels of anxiety } & \multicolumn{2}{|c|}{$\begin{array}{c}\text { Levels of } \\
\text { depression }\end{array}$} \\
\hline & Boys \% & Girls \% & Boys \% & Girls \% \\
\hline No anxiety/depression & 50.35 & 49.65 & 50.35 & 49.65 \\
\hline $\begin{array}{l}\text { Subthreshold- anxiety/ } \\
\text { depression }\end{array}$ & 38.24 & 61.76 & 38.24 & 61.76 \\
\hline Full anxiety/depression & 24.07 & 75.93 & 24.07 & 75.93 \\
\hline
\end{tabular}

$N=12,395$.

and girls respectively). When using a categorical approach, a strong association was also found between levels of anxiety and depression $\left(\chi^{2}(4)=3,807.565, p<.001, \gamma=.682\right)$ (Table 2).

\section{Psychopathology}

SDQ total score was found to be strongly related to both SAS $(r=.513, p<.001$ and $.619, p<.001$ for boys and girls respectively) and BDI-II scores $(r=.542, p<.001$ and $.654, p<.001$ for boys and girls respectively). After adjusting for the scores of depression/anxiety, associations between SDQ total score and SAS score $(r=.287$, $p=.004$ and $.302, p<.001$ for boys and girls respectively), as well as between SDQ and BDI-II score $(r=.346, p<.001$ and $.396, p<.001$ for boys and girls respectively) decreased, but remained significant, showing small to medium effect.

Descriptive statistics, as well as percentages of borderline and elevated scores of SDQ scales among groups of nondepressed/nonanxious, subthresholddepressed/anxious and depressed/anxious are shown in Table 3.

Controlling for age and BDI-II score, mean SDQ problem score differed significantly across the nonanxious, subthreshold-anxious and anxious groups for both genders $\left(F(2)=136.154, p<.001, \eta^{2}=.047\right.$ for boys and $F(2)=213.552, p<.001, \eta^{2}=.059$ for girls). All pair-wise post hoc comparisons were significant $(p<.001)$, indicating that mean scores of subthresholdanxious adolescents were higher than the mean scores of the nonanxious group (Cohen's $d=.920$ and .994 for boys and girls respectively), but lower than the mean scores of the anxious group (Cohen's $d=.839$ and 1.102 for boys and girls respectively).

Table 2 Percents of levels of anxiety and depression

\begin{tabular}{lcrrrr}
\hline & & \multicolumn{3}{c}{ Levels of Anxiety } & \\
\cline { 3 - 5 } & & NonA \% & SubA \% & A \% & Totals \\
\hline Levels of & NonD \% & 46.32 & 13.39 & .60 & 60.31 \\
depression & SubD \% & 14.75 & 12.98 & 1.46 & 29.19 \\
& D \% & 1.11 & 5.61 & 3.77 & 10.50 \\
Totals & & 62.18 & 31.98 & 5.83 & 100.00 \\
\hline
\end{tabular}

$\chi^{2}(4)=3,807.565 p<.001 ; \gamma=.682$.

$N=12,395$. NonA, nonanxious group; SubA, subthresholdanxious group; A, anxious group; NonD, nondepressed group; SubD, subthreshold-depressed group; D, depressed group.
Analysis resulted in a significant effect of group membership across levels of depression on SDQ total score for both genders $(F(2)=190.553, p<.001$, $\eta^{2}=.064$ for boys and $F(2)=292.788, \quad p<.001$, $\eta^{2}=.079$ for girls). Nondepressed adolescents had significantly lower scores than subthreshold-depressed adolescents $(p<.001$, Cohen's $d=.632$ and .776 for boys and girls respectively), whereas subthreshold-depressed adolescents had significantly lower scores then depressed adolescents $(p<.001$, Cohen's $d=1.126$ and 1.171 for boys and girls respectively).

\section{Functional impairment}

Logistic regression analysis revealed a significant effect of age $(\mathrm{OR}=1.219 ; 95 \% \mathrm{CI}=1.171-1.269)$ on dummycoded SDQ impact score as dependent $(0=$ no impairment; 1 = borderline/elevated scores), indicating that for each year of increase in age increases the probability of having functional impairments with $21.9 \%$. Gender also had a significant effect $(\mathrm{OR}=.726$; 95\% CI $=.675-$ .780 ), indicating that boys were predicted to have functional impairment with lower probability than girls.

Adjusting for the effect of age, gender and BDI-II score, the odds for a subthreshold-anxious adolescent having functional impairment was $1.795 \quad(95 \%$ $\mathrm{CI}=1.638-1.967)$ times greater than the odds for a nonanxious adolescent. Similarly, anxious adolescents were predicted to have functional impairment with a $2.519(95 \% \mathrm{CI}=1.982-3.201)$ times greater probability than their nonanxious counterparts $\left(\chi^{2}(5)=2845.482\right.$, $p<.001, \quad$ Nagelkerke- $R^{2}=.276, \quad$ AUC $=.773, \quad 95 \%$ $\mathrm{CI}=.776-.780)$.

In the second logistic regression model, when the effect of age, gender and SAS score were controlled, there were significant main effects of being subthreshold-depressed $(\mathrm{OR}=1.960 ; 95 \% \quad \mathrm{CI}=1.795-2.140)$ and depressed $(\mathrm{OR}=4.102 ; 95 \% \mathrm{CI}=3.455-4.871)$ on having functional impairment $\left(\chi^{2}(5)=2589.091, p<.001\right.$, Nagelkerke- $\left.R^{2}=.254, \mathrm{AUC}=.756,95 \% \mathrm{CI}=.749-.764\right)$.

\section{Suicidality}

Descriptive statistics of PSS and percentages of positive responders, by item, in nonanxious/nondepressed, subthreshold-anxious/depressed and anxious/depressed groups are reported in Table 4.

A significant effect of age $(O R=1.174, \quad 95 \%$ $\mathrm{CI}=1.126-1.225)$ was found when using dummy-coded PSS total score ( 0 or greater) as dependent in the logistic regression model, indicating that for each year increase on age increases the probability of suicidality with $17.4 \%$. Gender had also a significant effect on the dependent variable $(\mathrm{OR}=.546,95 \% \mathrm{CI}=.506-.590)$, indicating that boys were predicted to have suicidal thoughts/ideations with lower probability than girls.

Adjusting for the effect of age, gender and BDI-II score, the odds for a subthreshold-anxious adolescent for having suicidal thoughts/ideations were 1.788 (95\% $\mathrm{CI}=1.622-1.971)$ times greater than the odds for a nonanxious adolescent. Similarly, anxious adolescents were predicted to have suicidal thoughts /ideations with a $2.756(95 \% \mathrm{CI}=2.159-3.518)$ times greater probability than their nonanxious counterparts $\left(\chi^{2}(5)=3739.359\right.$, 
Table 3 Mean scores, standard deviations, as well as percentages of borderline and elevated scores of SDQ scales among groups of nondepressed/nonanxious, subthreshold-depressed/anxious and depressed/anxious.

\begin{tabular}{|c|c|c|c|c|c|c|c|}
\hline \multirow[b]{2}{*}{ SDQ } & \multirow[b]{2}{*}{ Total sample } & \multicolumn{3}{|c|}{ Levels of anxiety } & \multicolumn{3}{|c|}{ Levels of depression } \\
\hline & & NonA & SubA & A & NonD & SubD & $\mathrm{D}$ \\
\hline \multicolumn{8}{|l|}{ Problem scale } \\
\hline Borderline \% & 11.7 & 5.1 & 20.6 & 33.3 & 5.5 & 16.6 & 33.4 \\
\hline Elevated \% & 5.7 & 1.5 & 8.6 & 34.9 & 1.5 & 5.5 & 30.0 \\
\hline$M(S D)$ & $10.74(5.10)$ & $8.89(4.22)$ & 13.07 (1.80) & $17.77(4.76)$ & $\begin{array}{r}8.97 \\
(4.30)\end{array}$ & $12.45(4.46)$ & $17.07(4.57)$ \\
\hline \multicolumn{8}{|l|}{ Impact scale } \\
\hline Borderline \% & 4.2 & 4.8 & 3.0 & 1.1 & 5.1 & 3.6 & 1.4 \\
\hline Elevated \% & 37.2 & 23.9 & 55.3 & 83.6 & 23.7 & 49.1 & 82.0 \\
\hline Median (Interquartile Range) & $0(0-3)$ & $0(0-1)$ & $2(0-5)$ & $6(3-10)$ & $0(0-1)$ & $1(0-4)$ & $6(3-10)$ \\
\hline
\end{tabular}

$N=12,395$. SDQ, Strength and Difficulties Questionnaire; NonA, nonanxious group; SubA, subthreshold-anxious group; A, anxious group; NonD, nondepressed group; SubD, subthreshold-depressed group; D, depressed group.

Table 4 Descriptive statistics of PSS and per cent of positive responders by item in nondepressed/nonanxious, subthresholddepressed/anxious and depressed/anxious groups

\begin{tabular}{|c|c|c|c|c|c|c|c|}
\hline & \multirow[b]{2}{*}{ Total sample } & \multicolumn{3}{|c|}{ Levels of anxiety } & \multicolumn{3}{|c|}{ Levels of depression } \\
\hline & & NonA & SubA & A & NonD & SubD & $\mathrm{D}$ \\
\hline PSS Median (Intequartile Range) & $0(0-1)$ & $0(0-0)$ & $1(0-3)$ & $4(1-10)$ & $0(0-0)$ & $0(0-2)$ & $4(2-9)$ \\
\hline Life not worth living during past 2 weeks $\%$ & 27.00 & 14.78 & 41.95 & 75.29 & 12.46 & 38.93 & 77.35 \\
\hline Wish that were dead during past 2 weeks $\%$ & 18.52 & 8.21 & 30.00 & 65.61 & 6.99 & 25.08 & 66.52 \\
\hline Thought of taking own life during past 2 weeks $\%$ & 16.79 & 7.86 & 26.74 & 57.50 & 6.74 & 21.76 & 60.70 \\
\hline
\end{tabular}

$N=12,395$. PSS, Paykel Suicide Scale; NonA, nonanxious group; SubA, subthreshold-anxious group; A, anxious group; NonD, nondepressed group; SubD, subthreshold-depressed group; D, depressed group.

$p<.001, \quad$ Nagelkerke- $R^{2}=.361, \quad$ AUC $=.812, \quad 95 \%$ $\mathrm{CI}=.805-.819)$.

When assessing the effect of levels of depression on suicidality, we found significant main effects of being subthreshold-depressed $(\mathrm{OR}=3.065 ; 95 \% \mathrm{CI}=2.792-$ $3.364)$ and depressed $(\mathrm{OR}=9.210 ; 95 \% \mathrm{CI}=7.700$ 11.016) when the effect of age, gender and SAS scores were controlled for $\chi^{2}(5)=3492,978, p<.001$, Nagelkerke- $R^{2}=.340$, AUC $\left.=.800,95 \% \mathrm{CI}=.793-.807\right)$

\section{Discussion}

Similar to the study of Angst et al. (1997) on a community sample of individuals ages 19-20, in the current study approximately half of the adolescents met the criteria for threshold and/or subthresholddepression and/or anxiety. Although our focus was on subthreshold-depression and subthreshold-anxiety, it is noteworthy that based on screening tools an exceptionally high proportion of this sample was categorized as depressed $(10.5 \%)$ and anxious (5.8\%). Similar to prior findings, our results show a high prevalence of subthreshold-depression and anxiety among adolescents throughout Europe (Fergusson et al., 2005; Wittchen et al., 1998). According to our data, almost one third of adolescents had current subthreshold-depression and one third had current subthreshold-anxiety. Importantly, even these less severe cases were associated with elevated levels of psychopathology, and increased risk for functional impairment and suicidality.

No difference in the prevalence of depression among preadolescent boys and girls has been described (Anderson, Williams, McGee, \& Silva, 1987; Kashani et al., 1983). After ages 11-13 and throughout adulthood, this trend changes and female subjects are approximately twice as likely as male subjects to be depressed and the same prevalence estimate is true for anxiety disorders (Angold, Costello, \& Worthman, 1998; Mackinaw-Koons \& Vasey, 2000). In the current study, we observed similar gender distributions in the depressed and anxiety groups and in the subthreshold-depressed and subthreshold-anxiety groups as well.

In this large international sample, we found a strong correlation between depression according to BDI-II and anxiety according to SAS. While high comorbidity of both threshold and subthresholddepression and anxiety was expected (Kessler et al., 2005; Unick et al., 2009; Wittchen et al., 1994), it is still surprising that only one tenth of all adolescents with threshold-depression or threshold-anxiety had 'pure forms' of the disorders. The presence of comorbid (even subthreshold) MDE and anxiety is associated with more severe psychopathology, greater impairment, increased suicidality and worse outcome than in noncomorbid conditions (Altamura, Montresor, Salvadori, \& Mundo, 2004; Foley et al., 
2006; Guberman \& Manassis, 2011). Our results highlight the importance of assessing comorbidity of depression and anxiety in adolescents. In contrast to the high comorbidity of threshold psychopathology, the percentage of pure forms of subthresholddepression and subthreshold-anxiety were found to be much higher (up to 40-50).

Based on both dimensional and categorical diagnostic models, our data revealed a strong association between the SDQ total score and SAS/BDI scores. According to the SDQ Impact scale, after adjusting for age, gender and SAS scores, being subthresholddepressed increased the probability of having functional impairment, the odds for having functional impairment for depressed was four times more than being nondepressed. Similarly, adjusting for the effects of age, gender and BDI-II score, adolescents with both subthreshold-anxiety and threshold-anxiety showed greater probability of having functional impairment than nonanxious adolescents. The elevated level of psychopathology and the increased risk of functional impairment suggest that adolescents with subthreshold-depression and with subthreshold-anxiety already have clinically meaningful symptoms, requiring professional intervention.

Gender here had a significant effect on suicide behaviour, similar to earlier findings (Wunderlich, Bronisch, Wittchen, \& Carter, 2001). According to the PSS total score, subthreshold conditions increased the probability of having suicidal thoughts / ideations, and the odds for having suicidal thoughts/ideations in a full syndrome condition were even greater in both anxiety and depression. Namely, after adjusting for age, gender and BDI-II scores, the odds for a subthreshold-anxious adolescent to have suicidal thoughts/ideations were approximately two times greater than the odds for a nonanxious adolescent, and being anxious increased the probability of having suicidal thoughts / ideations almost two and a half times. We found the same pattern in the case of depression: adjusting for age, gender and SAS score, adolescents with subthreshold-depression showed three times greater probability of having suicidal thoughts/ideations than nonanxious adolescents, whereas being threshold-depressed increased the probability of having suicidal thoughts/ideations nine times more than being nondepressed. These data indicate that both subthreshold and threshold forms of depression increase the risk of having suicidal thoughts/ ideations, even more than subthreshold and threshold-anxiety. From a clinical standpoint, early recognition and intervention of subthresholddepression and subthreshold-anxiety may prevent full-onset depression/anxiety and significantly reduce the related suicide risk.

This study's results contribute to the current discussions about categorical and dimensional systems. We used a dimensional diagnostic model of depression and anxiety and introduced a third con- dition, subthreshold-depression/subthreshold-anxiety, based on classical categorical approaches. Our findings, that the level of risk for increased burden and suicide among adolescents with subthresholddepression/anxiety is between the risk for those nondepressed/nonanxious and depressed/anxious, supports the suggestion of previous studies that show that subthreshold and full DSM-IV-TR depression/anxiety could be on the same continuum (Fergusson et al., 2005; Klein et al., 2009; Lewinsohn et al., 2000; Shankman et al., 2009). Based on our data, inputting subthreshold-depression and subthreshold-anxiety into the diagnostic systems could provide an important bridge between traditional categorical diagnostic approaches and dimensional models.

Limitations of these findings include their being cross-sectional. Longitudinal studies are needed to understand the potential negative sequel of subthreshold-depression and anxiety. As this study took place in eleven European countries, cross-cultural differences must also be taken into account. Finally, our data are based on self-report, which can be biased.

In conclusion, our study supports the dimensional rather than the categorical nature of adolescent subthreshold and full syndrome depression and anxiety. It highlights the importance of early detection especially, as it may be associated with suicidal behaviour. Recognition and appropriate intervention for adolescent subthreshold conditions may significantly save lives of young people.

\section{Acknowledgements}

The SEYLE project is supported through Coordination Theme 1 (Health) of the European Union Seventh Framework Program (FP7), Grant agreement number HEALTH-F2-2009-223091.

The authors were independent of the funders in all aspects of study design, data analysis and writing of this manuscript. The Project Leader and Coordinator of the SEYLE project is Professor in Psychiatry and Suicidology Danuta Wasserman, Karolinska Institutet (KI), Head of the National Centre for Suicide Research and Prevention of Mental Ill-Health and Suicide (NASP), at KI, Stockholm, Sweden. Other members of the Executive Committee are Professor Marco Sarchiapone, Department of Health Sciences, University of Molise, Campobasso, Italy; Vladimir Carli, National Centre for Suicide Research and Prevention of Mental Ill-Health (NASP), Karolinska Institutet, Stockholm, Sweden; Professor Christina Hoven and Anthropologist Camilla Wasserman, Department of Child and Adolescent Psychiatry, New York State Psychiatric Institute, Columbia University, New York, USA. The SEYLE Consortium comprises centres in 12 European countries. Site leaders for each respective centre and country are: Danuta Wasserman (NASP, Karolinska 
Institutet, Sweden, Coordinating Centre), Christian Haring (University for Medical Information Technology, Austria), Airi Varnik (Estonian-Swedish Mental Health \& Suicidology Institute, Estonia), Jean-Pierre Kahn (University of Nancy, France), Romuald Brunner (University of Heidelberg, Germany), Judit Balazs (Vadaskert Child and Adolescent Psychiatric Hospital, Hungary), Paul Corcoran (National Suicide Research Foundation, Ireland), Alan Apter (Schneider Children's Medical Centre of Israel, Tel-Aviv University, Tel Aviv, Israel), Marco Sarchiapone (University of Molise, Italy), Doina Cosman (Iuliu Hatieganu University of Medicine and Pharmacy, Romania), Vita Postuvan (University of Primorska, Slovenia) and Julio Bobes (University of Oviedo, Spain).
The Psychiatric Clinic of the University Basel hosted the Workshop: Theory meets Practice - Ethical Issues in Research with Minors and other Vulnerable Groups, 14.2.2012. It was supported by grant, through an accompanying project on research ethics funded by the Botnar Foundation, Basel (Project Leader: Prof. Dr. Stella Reiter-Theil).

All the authors have declared that they have no competing or potential conflicts of interest.

\section{Correspondence}

Judit Balázs M.D., Ph.D., Vadaskert Child and Adolescent Psychiatry Hospital, Huvosvolgyi ut 116, Budapest 1021, Hungary; Email: judit.agnes.balazs @gmail.com

\section{Key points}

- This study investigates the characteristics of adolescent subthreshold-depression and subthresholdanxiety in a large European sample, with a focus on suicidality.

- According to our data, both subthreshold-depression and subthreshold-anxiety are very prevalent, and associated with an increased burden of disease and suicidal risk.

- Our study highlights the importance of early detection of subthreshold-depression and subthresholdanxiety to reduce psychopathology and distress in adolescents, especially as it may be associated with suicidal behaviour.

- The current study supports the continuum, that is the dimensional rather than categorical nature of adolescent subthreshold and full syndrome depression and anxiety.

\section{References}

Altamura, A.C., Montresor, C., Salvadori, D., \& Mundo, E. (2004). Does comorbid subthreshold anxiety affect clinical presentation and treatment response in depression? A preliminary 12-month naturalistic study. The International Journal of Neuropsychopharmacology, 7, 481-487.

American Psychiatric Association. (2000). Diagnostic and statistical manual of mental disorders fourth edition text revised. Washington, DC: American Psychiatric Association.

Anderson, J.C., Williams, S., McGee, R., \& Silva, P.A. (1987). DSM-III disorders in preadolescent children: Prevalence in a large sample from the general population. Archives of General Psychiatry, 44, 69-76.

Angold, A., Costello, E.J., \& Worthman, C.M. (1998). Puberty and depression: The roles of age, pubertal status and pubertal timing. Psychological Medicine, 28, 51-61.

Angst, J., Merikangas, K.R., \& Preisig, M. (1997). Subthreshold syndromes of depression and anxiety in the community. Journal of Clinical Psychiatry, 58 (Supp1. 8), 6-10.

Balázs, J., Bitter, I., Lecrubier, Y., Csiszér, N., \& Ostorharics, Gy. (2000). Prevalence of subthreshold forms of psychiatric disorders in persons making suicide attempts in Hungary. European Psychiatry, 15, 354-361.

Beck, A.T., Steer, R.A., Ball, R., \& Ranieri, W. (1996). Comparison of Beck Depression Inventories -IA and -II in psychiatric outpatients. Journal of Personality Assessment, 67, 588-597.

Byrne, B.M., Stewart, S.M., \& Lee, P.W.H. (2004). Validating the Beck Depression Inventory-II for Hong Kong Community adolescents. International Journal of Testing, 4, 199-216.

Carter, R.M., Wittchen, H.U., Pfister, H., \& Kessler, R.C. (2001). One-year prevalence of subthreshold and threshold
DSM-IV generalized anxiety disorder in a nationally representative sample. Depression and Anxiety, 13, 78-88.

Cohen, J. (1988). Statistical power analysis for the behavioral sciences (2nd edn). Hillsdale, NJ: Erlbaum.

Fergusson, D.M., Horwood, L.J., Ridder, E.M., \& Beautrais, A.L. (2005). Subthreshold-depression in adolescence and mental health outcomes in adulthood. Archives of General Psychiatry, 62, 66-72.

Foley, D.L., Goldston, G.B., Costello, E.J., \& Angold, A. (2006). Proximal psychiatric risk factors for suicidality in youth. The Great Smoky Mountains Study. Archives of General Psychiatry, 63, 1017-1024.

Goodman, R., Meltzer, H., \& Bailey, V. (1998). The Strengths and Difficulties Questionnaire: A pilot study on the validity of the self-report version. European Child \& Adolescent Psychiatry, 7, 125-130.

Gould, M.S., King, R., Greenwald, S., Fisher, P., SchwabStone, M., Kramer, R., .. \& Shaffer, D. (1998). Psychopathology associated with suicidal ideation and attempts among children and adolescents. Journal of the American Academy of Child and Adolescent Psychiatry, 37, 915923.

Guberman, C., \& Manassis, K. (2011). Symptomatology and family functioning in children and adolescents with comorbid anxiety and depression. Journal of the Canadian Academy of Child and Adolescent Psychiatry, 20, 186-195.

Helmchen, H., \& Linden, M. (2000). Subthreshold disorders in psychiatry: Clinical reality, methodological artifact and double-threshold problem. Comprehensive Psychiatry, 41 (Suppl. 1), 1-7.

Johnson, J., Weissman, M.M., \& Klerman, G.L. (1992). Service utilization and social morbidity associated with depressive symptoms in the community. Journal of the American Medical Association, 267, 1478-1483. 
Judd, L.L., Rapaport, M.H., Paulus, M.P., \& Brown, J.L. (1994). Subsyndromal symptomatic depression: A new mood disorder?. Journal of Clinical Psychiatry, 55 (Suppl.), 18-28.

Karsten, J., Nolen, W.A., Penninx, B.W., \& Hartman, C.A (2011). Subthreshold-anxiety better defined by symptom self-report than by diagnostic interview. Journal of Affective Disorders, 129, 236-243.

Kashani, J.H., McGee, R.D., Clarkson, S.E., Anderson, J.C., Walton, L.E., Williams, S., ... \& McKnew, D.H. (1983). Depression in a sample of nine-year-old children. Archives of General Psychiatry, 40, 1217-1223.

Keenan, K., Hipwell, A., Feng, X., Babinski, D., Hinze, A., Rischall, M., \& Henneberger, A. (2008). Subthreshold symptoms of depression in preadolescent girls are stable and predictive of depressive disorders. Journal of the American Academy of Child and Adolescent Psychiatry, 47, 14331442 .

Kertz, S.J., \& Woodruff-Borden, J. (2011). Human and economic burden of GAD, subthreshold GAD, and worry in a primary care sample. Journal of Clinical Psychology in Medical Settings, 18, 281-290.

Kessler, R.C., Chiu, W.T., Demler, O., Merikangas, K.R., \& Walters, E.E. (2005). Prevalence, severity, and comorbidity of 12-month DSM-IV disorders in the National Comorbidity Survey Replication. Archives of General Psychiatry, 62, 617627.

Klein, D.N., Shankman, S.A., Lewinsohn, P.M., \& Seeley, J.R. (2009). Subthreshold depressive disorder in adolescents: Predictors of escalation to full-syndrome depressive disorders. Journal of the American Academy of Child and Adolescent Psychiatry, 48, 703-710.

Kramer, A.A., \& Zimmerman, J.E. (2007). Assessing the calibration of mortality benchmarks in critical care: The hosmer-lemeshow test revisited. Critical Care Medicine, 35, 2052-2056.

Lecrubier, Y. (2008). Refinement of diagnosis and disease classification in psychiatry. European Archives of Psychiatry and Clinical Neuroscience, 258 (Suppl. 1), 6-11.

Lewinsohn, P.M., Solomon, A., Seeley, J.R., \& Zeiss, A. (2000). Clinical implications of "subthreshold" depressive symptoms. Journal of Abnormal Psychology, 109, 345-351.

Mackinaw-Koons, B., \& Vasey, M.W. (2000). Considering sex differences in anxiety and its disorders across the life span: A construct-validation approach. Applied and Preventive Psychology, 9, 191-209.

McDowell, I. (2006). Measuring health: A guide to rating scales and questionnaires (3rd edn). Oxford: Oxford University Press.

Möller, H.J. (2008). Systematic of psychiatric disorders between categorical and dimensional approaches: Kraepelin's dichotomy and beyond. European Archives of Psychiatry and Clinical Neuroscience, 258 (Suppl. 2), 48-73.

Nauta, M.H., Festen, H., Reichart, C.G., Nolen, W.A., Stant, A.D., Bockting, C.L., ... \& de Vries, S.O. (2012). Preventing mood and anxiety disorders in youth: A multi-centre RCT in the high risk offspring of depressed and anxious patients. BMC Psychiatry, 12, 31

Okasha, A. (2009). Would the use of dimensions instead of categories remove problems related to subthreshold disor- ders?. European Archives of Psychiatry and Clinical Neuroscience, 259 (Suppl. 2), 129-133.

Paykel, E.S., Myers, J.K., Lindenthal, J.J., \& Tanner, J. (1974). Suicidal feelings in the general population: A prevalence study. The British Journal of Psychiatry, 124, 460-469.

Pine, D.S., Cohen, E., Cohen, P., \& Brook, J. (1999). Adolescent depressive symptoms as predictors of adult depression: Moodiness or mood disorder?. American Journal of Psychiatry, 156, 133-135.

Rubin, D.B. (1987). Multiple imputation for nonresponse in surveys. New York: J. Wiley \& Sons.

Rucci, P., Gherardi, S., Tansella, M., Piccinelli, M., Berardi, D., Bisoffi, G., ... \& Pini, S. (2003). Subthreshold psychiatric disorders in primary care: Prevalence and associated characteristics. Journal of Affective Disorders, 76, 171-181.

Shankman, S.A., Lewinsohn, P.M., Klein, D.N., Small, J.W., Seeley, J.R., \& Altman, S.E. (2009). Subthreshold conditions as precursors for full syndrome disorders: A 15-year longitudinal study of multiple diagnostic classes. Journal of Child Psychology and Psychiatry, 50, 1485-1494.

SPSS, Inc. (2011). SPSS: Version 20.0 for windows. Chicago: SPSS, Inc.

Unick, G.J., Snowden, L., \& Hastings, J. (2009). Heterogeneity in comorbidity between major depressive disorder and generalized anxiety disorder and its clinical consequences. The Journal of Nervous and Mental Disease, 197, 215-224.

Vostanis, P. (2006). Strengths and difficulties questionnaire: Research and clinical applications. Current Opinion in Psychiatry, 19, 367-372.

Wasserman, D., Carli, V., Wasserman, C., Apter, A., Balazs, J., Bobes, J., ... \& Hoven, C.W. (2010). Saving and Empowering Young Lives in Europe (SEYLE): A randomized controlled trial. BMC Public Health, 10, 192.

Wittchen, H.U., Nelson, C.B., \& Lachner, G. (1998). Prevalence of mental disorders and psychosocial impairments in adolescents and young adults. Psychological Medicine, 28, 109126 .

Wittchen, H.-U., Zhao, S., Kessler, R.C., \& Eaton, W.W. (1994). DSM- Ill-R generalized anxiety disorder in the National comorbidity Survey. Archives of General Psychiatry, 51, 355364

World Health Organization. (1992). The ICD-10 classification of mental and behavioral disorders. Geneva: World Health Organization.

Wunderlich, U., Bronisch, T., \& Wittchen, H.U. (1998). Comorbidity patterns in adolescents and young adults with suicide attempts. European Archives of Psychiatry and Clinical Neuroscience, 248, 87-95.

Wunderlich, U., Bronisch, T., Wittchen, H.U., \& Carter, R. (2001). Gender differences in adolescents and young adults with suicidal behaviour. Acta Psychiatrica Scandinavica, 104, 332-339

Zung, W.W. (1971). A rating instrument for anxiety disorders. Psychosomatics, 12, 371-379.

Accepted for publication: 3 September 2012

Published online: 18 January 2013 